\title{
A RESPIRAÇÃO ORAL EM CRIANÇAS E SUAS REPERCUSSÕES NO ESTADO NUTRICIONAL
}

\author{
Mouth breathing in children and its repercussions in the \\ nutritional state
}

\author{
Daniele Andrade da Cunha ${ }^{(1)}$, Giselia Alves Pontes da Silva ${ }^{(2)}$, Maria Eugênia Farias Almeida \\ Motta $^{(3)}$, Cybelle Rolim de Lima ${ }^{(4)}$, Hilton Justino da Silva ${ }^{(5)}$
}

\begin{abstract}
RESUMO
Objetivo: identificar o estado nutricional, função mastigatória, aeração nasal, dados sobre o sono, olfato e paladar de crianças respiradoras orais e compará-las com um grupo de crianças respiradoras nasais. Métodos: avaliou-se estado nutricional, modo respiratório e mastigação de 77 crianças respiradoras orais e 154 respiradoras nasais entre seis e dez anos. Resultados: os respiradores orais apresentaram alterações no padrão mastigatório, quando comparados aos respiradores nasais, porém não houve diferença estatisticamente significante em relação ao estado nutricional. Conclusão: em crianças respiradoras orais houve maior alteração no sono, diminuição da ingestão de carboidratos e aumento da ingestão de lipídeos, porém não foram encontrados dados consistentes que apontem a associação entre a respiração oral e o estado nutricional.
\end{abstract}

DESCRITORES: Respiração Bucal; Estado Nutricional; Fonoaudiologia

\section{INTRODUÇÃO}

As doenças respiratórias na infância abrangem amplo espectro de eventos mórbidos de diferentes etiologias e gravidade; caracterizam-se por comprometer uma ou mais porções do trato respiratório da criança. A presença de obstrução nas vias aéreas superiores leva o paciente a respirar pela boca ${ }^{1}$. 0 indivíduo passa a utilizar a cavidade oral como um

(1) Fonoaudióloga; Coordenadora do Curso de Fonoaudiologia da Faculdade Integrada do Recife; Mestre e doutoranda em Nutrição pela Universidade Federal de Pernambuco.

(2) Médica; Professora Adjunto de Pediatria da Universidade Federal de Pernambuco; Doutora em Pediatria pela Escola Paulista de Medicina.

(3) Médica; Professora Adjunto de Pediatria da Universidade Federal de Pernambuco; Mestre em Saúde da Criança e do Adolescente pela Universidade Federal de Pernambuco; Doutora em Pediatria e Ciências Aplicadas à Pediatria pela Escola Paulista de Medicina.

(4) Nutricionista; Mestranda em Nutrição pela Universidade Federal de Pernambuco.

(5) Fonoaudiólogo; Docente da Universidade Federal de Pernambuco e Faculdade Integrada do Recife; Mestre em Morfologia pela Universidade Federal de Pernambuco; Doutor em Nutrição pela Universidade Federal de Pernambuco. conduto passivo na respiração ${ }^{2}$, surgindo, assim, a respiração oral ${ }^{3}$, que poderá se tornar crônica e, a partir daí, surgem manifestações clínicas em diversos aparelhos e sistemas, caracterizando o quadro de respiração oral ${ }^{2}$.

A respiração oral tem como conseqüência a adaptação de toda a musculatura facial que provoca modificações nas arcadas dentárias e no posicionamento dos dentes, acarretando alterações estruturais na face, incluindo lábios, língua, palato e mandíbula, que se adaptarão ao novo padrão respiratório ${ }^{4}$. Outras características de crianças respiradoras orais são o cansaço freqüente, sonolência diurna, adinamia, apetite reduzido, alterações nutricionais, enurese noturna e déficit de aprendizado ${ }^{5}$.

O respirador oral geralmente apresenta olfato e paladar diminuídos. Assim, a opção pelo tipo de alimento não é feita pelo apetite, mas pela consistência e facilidade de ingestão, permitindo que o paciente continue respirando pela boca ${ }^{3}$. Poucas são as explicações para justificar a mudança do peso corporal do indivíduo respirador oral. Algumas delas relacionam-se às mudanças no processo mastigatório e à dificuldade de deglutição, causadas pela obstrução faríngea, nasal ou palatina; outra, refere-se à diminuição do olfato pela obstrução nasal crônica, alterando o paladar ${ }^{6}$. 
Embora os estudos mostrem possível relação entre respiração oral e mudanças no processo alimentar, com conseqüente interferência no estado nutricional, observam-se poucos estudos nacionais acerca dessas alterações, tornando-se relevante a sua investigação.

Assim, o presente estudo teve como objetivo geral identificar o estado nutricional, e como objetivos específicos identificar as características da mastigação, modo respiratório, dados sobre o sono, olfato e paladar de crianças com respiração oral e comparar os resultados com um grupo de crianças respiradoras nasais.

\section{MÉTODOS}

Esta pesquisa foi realizada nos Ambulatórios de Alergologia e Pediatria do Hospital das Clínicas da Universidade Federal de Pernambuco e do Hospital Barão de Lucena, conveniados ao Sistema Único de Saúde (SUS). A população de estudo foi composta por 77 crianças respiradoras orais e 154 respiradoras nasais, na faixa etária de seis a dez anos, atendidas no período de abril a setembro de 2004.

Inicialmente, os responsáveis assinaram o termo de consentimento livre e esclarecido autorizando a participação da criança no estudo. A seguir, foi solicitado aos acompanhantes das crianças que respondessem a um formulário especificamente elaborado para esta pesquisa, fornecendo dados sobre condições socioeconômicas, alimentação, mastigação, olfato, paladar e aspectos do sono das crianças. Por fim, as crianças passaram por uma avaliação fonoaudiológica e nutricional.

Realizou-se estudo exploratório do tipo transversal, comparando crianças que apresentavam respiração oral com crianças respiradoras nasais. Para formar o grupo de casos, foram incluídas crianças portadoras de respiração oral secundária à rinite alérgica, confirmada em prontuário médico. Foi formado um grupo controle, composto por crianças com respiração nasal e que não tinham rinite alérgica.

A amostra foi de conveniência, estimou-se uma amostra mínima de 67 respiradores orais e 134 respiradores nasais.

Foram excluídas, nos dois grupos, as crianças em uso de aparelho ortodôntico, portadoras de doenças sistêmicas, anormalidades craniofaciais, distúrbios neurológicos, asma, hipertrofias de adenóides e/ou de amígdalas.

As variáveis estudadas para a caracterização da amostra foram socioeconômicas (chefe da família, fonte da água, sanitário, renda mensal), maternas (idade, escolaridade, trabalho materno fora do lar, quantidade de filhos), criança (idade, gênero, duração do aleitamento materno, ronco noturno e presença de saliva no travesseiro).
As variáveis fonoaudiológicas foram: modo respiratório (caracterizado por saída de ar uni ou bilateral), características e avaliação da mastigação (ruidosa, lábios fechados durante a mastigação, predominância do lado da mastigação, escape de comida e tempo de mastigação) e dados questionados ao acompanhante sobre o olfato, o paladar e o sono da criança (caracterizado por presença ou não dessas queixas).

O estado nutricional foi avaliado através do IMC, calculado pela razão entre peso corporal $(\mathrm{Kg})$ e estatura $(\mathrm{cm})$ ao quadrado. Os valores obtidos foram comparados com o padrão do NCHS (National Center for Health Statistics) e utilizaram-se os seguintes pontos de corte: baixo peso < P5, eutrófico P5 até < $\mathrm{P} 85$, risco de sobrepeso e sobrepeso/obesidade $=$ P85. O índice estatura/idade também foi utilizado como indicador do estado nutricional, expresso em escore Z, utilizando a curva do NCHS como referência. O retardo de crescimento linear foi diagnosticado nas crianças com o índice estatura/idade $<-2$ escore $z$, estando em risco para retardo de crescimento linear aquelas entre -2 e - 1 escore $Z$.

Na obtenção da medida da aeração nasal foi utilizado o Espelho Nasal Milimetrado de Altmann. Foram seguidas as instruções (modo de usar e recomendações) propostas na embalagem padrão do produto, bem como no site de divulgação do produto (www.profono.com.br). A avaliação foi realizada de acordo com os seguintes procedimentos: sem interferência de ar-condicionado no ambiente, o examinador na posição de pé e o sujeito na posição sentada, com a cabeça reta. Encostou-se o espelho abaixo do nariz, centralizado na altura da espinha nasal anterior. Marcou-se a região embaçada pelo ar expirado com caneta de retroprojetor azul no próprio espeIho, primeiro a narina direita e em seguida a esquerda. Realizou-se a transferência da marcação da aeração obtida do espelho para uma folha do Bloco de Referência, posicionando-a sobre o espelho e copiando o traçado de forma direta, por transparência.

O protocolo para avaliação da mastigação foi elaborado por uma das pesquisadoras, adaptado ${ }^{7,8}$. A criança sentou-se em uma cadeira e foi solicitado que comesse normalmente um pão francês de $25 \mathrm{~g}$. As crianças foram filmadas durante a mastigação do pão com uma filmadora digital Sony Digital Hand Cam VCR TRV 130 NTSC, fixada em um tripé com distância de um metro e meio; os dados foram gravados em fita $8 \mathrm{~mm}$. Paralelo ao procedimento de filmagem, o tempo da mastigação foi registrado com um cronômetro da marca Casio. Após a análise das fitas, a mastigação foi caracterizada segundo a presença ou ausência de mastigação ruidosa, lábios fechados, predominância por lado de mastigação (uni ou bilateral), escape de comida no momento da mastigação e tempo da mastigação. 
Na avaliação antropométrica, foi realizada a pesagem das crianças descalças, usando apenas trajes íntimos, utilizando-se balança eletrônica portátil (Filizola) com escala de $100 \mathrm{~g}$ (para as crianças com mais de $15 \mathrm{~kg}$ e acima de dois anos de idade). Para avaliar a estatura, as crianças se posicionaram de forma ereta, com os pés unidos, descalços e no chão, olhar para diante, membros superiores laterais ao tronco, de costas para a área escolhida como marcador. A aferição da estatura foi feita com a régua antropométrica de $200 \mathrm{~cm}$ acoplada à balança. O valor encontrado foi expresso em centímetros.

Todas as informações foram armazenadas no banco de dados elaborado no programa de estatística EpiInfo, versão 6.04, com dupla entrada, checagem e validação da digitação. Na análise estatística, foram utilizados o teste de qui-quadrado de Pearson, com correção de Yates, ou o teste exato de Fisher, quando necessário, para a análise univariada das variáveis categóricas. Para as variáveis contínuas foi verificado se havia distribuição normal e realizado o teste de Bartlett para verificar a homogeneidade da variância. Os resultados foram expressos como mediana (percentis 25 e 75), sendo utilizado o teste de MannWhitney (não paramétrico) devido a não homogeneidade dos dados. Admitiu-se nível de confiança de $5 \%(p=0,05)$ para aceitação de associação.

Esta pesquisa foi aprovada pelo Comitê de Ética em Pesquisa com Seres Humanos da Universidade Federal de Pernambuco, sob o protocolo de número 062/2004.

O cálculo do tamanho amostral foi realizado por meio do programa statcalc do software Epi-Info, versão 6.04 do CDC (Center for Disease Control), utilizando a opção para estudo transversal. Considerando o nível de confiança de $90 \%$, o poder da amostra de $80 \%$, a freqüência de sobrepeso (índice de massa corporal $(\mathrm{IMC})>$ percentil $(\mathrm{P})$ 85) nas crianças respiradoras nasais de $10 \%{ }^{9}$, uma freqüência estimada de sobrepeso (IMC > P85) no grupo de respiradores orais de $25 \%$, na proporção de 1:2.

\section{RESULTADOS}

Foram incluídas neste estudo 231 crianças, sendo 77 (33,3\%) com respiração oral e 154 (66,7\%) respiradores nasais. Não houve diferença estatística entre os grupos quanto à distribuição por idade, gênero e duração do aleitamento materno (Tabela 1).

A Tabela 2 mostra os resultados da distribuição de freqüência das variáveis relacionadas ao olfato, paladar, mastigação e aeração nasal. Houve diferença estatisticamente significante entre os grupos nos seguintes aspectos da avaliação da mastigação: maior ocorrência de mastigação ruidosa ( $p<0,001)$, escape de comida $(p=0,03)$ e menor possibilidade de vedamento labial $(p=0,001)$ nas crianças com respiração oral. A saída de ar bilateral foi predominante nas crianças respiradoras orais e encontrada em todas as crianças respiradoras nasais, com diferença estatística entre os grupos ( $p<0,001)$.

O ronco noturno esteve presente em 73\% (54/75) dos respiradores orais e em 41,2\% (63/153) dos respiradores nasais $(p<0,001)$. A presença de saliva no travesseiro foi referida pelas mães em 73\% (54/74) dos respiradores orais e 47,7\% (73/153) dos respiradores nasais $(p<0,001)$.

Na amostra, não foi observada diferença estatística significante em relação à avaliação do estado nutricional ao se utilizar o IMC ( $p=0,24)$; também não foi encontrada diferença estatística quando utilizou-se o índice estatura/idade, $p=0,12$ (Tabela 3).

Tabela 1 - Distribuição da amostra segundo idade, gênero, duração do aleitamento materno

\begin{tabular}{ccc}
\hline VARIÁVEIS & RESPIRADORES & RESPIRADORES \\
& ORAIS & ESTAT ÍSTICA \\
\hline Idade & $\mathrm{N}(\%)$ & $\mathrm{N}(\%)$ \\
\hline 6 anos & $14(18,2 \%)$ & $32(20,8 \%)$ \\
\hline 7 anos & $19(24,7 \%)$ & $38(24,7 \%)$ \\
\hline 8 anos & $14(18,2 \%)$ & $27(17,5 \%)$ \\
\hline 9 anos & $16(20,7 \%)$ & $27(17,5 \%)$ \\
\hline 10 anos & $14(18,2 \%)$ & $p=0,971$
\end{tabular}




\begin{tabular}{cccc}
\hline Duração do aleitamento materno* & & \\
\hline$<4$ meses & $43(62,3 \%)$ & $80(60,6 \%)$ & \\
\hline$\geq 4$ meses & $26(37,7 \%)$ & $52(39,4 \%)$ & $p=0,933$ \\
\hline
\end{tabular}

*Nota: 69 respiradores orais e 132 respiradores nasais $<$ menor, $\geq$ maior ou igual

\section{Tabela 2 - Distribuição da amostra segundo variáveis relacionadas ao olfato, paladar,} mastigação e aeração nasal

\begin{tabular}{|c|c|c|c|}
\hline VARIÁVEIS & $\begin{array}{c}\text { RESPIRADORES } \\
\text { ORAIS }\end{array}$ & $\begin{array}{c}\text { RESPIRADORES } \\
\text { NASAIS }\end{array}$ & ESTATÍSTICA \\
\hline Dificuldade Sentir Cheiro & $7(9,5 \%)$ & $9(5,8 \%)$ & $p=0,47$ \\
\hline Dificuldade Sentir Gosto & $6(7,9 \%)$ & $4(2,6 \%)$ & $p=0,07$ \\
\hline Lentidão durante a mastigação & $49(63,6 \%)$ & $77(50 \%)$ & $p=0,07$ \\
\hline Uso de Líquido durante a mastigação & $65(85,5 \%)$ & $127(82,5 \%)$ & $p=0,69$ \\
\hline Prefere Alimentos Pastosos & $29(37,7 \%)$ & $51(33,1 \%)$ & $p=0,59$ \\
\hline Mastigação Ruidosa & $43(55,8 \%)$ & $43(27,9 \%)$ & $p<0,001$ \\
\hline Lábios Fechados & $43(55,8 \%)$ & $119(77,3 \%)$ & $p=0,001$ \\
\hline \multicolumn{4}{|l|}{ Lado Mastigatório } \\
\hline $\begin{array}{l}\text { Predominantemente Bilateral } \\
\text { Alternado }\end{array}$ & \multicolumn{2}{|c|}{ Alternado } & \\
\hline \multicolumn{4}{|l|}{ Unilateral } \\
\hline Escape de Comida & $14(18,2 \%)$ & $12(7,8 \%)$ & $p=0,03$ \\
\hline \multicolumn{4}{|l|}{ Tempo de Mastigação } \\
\hline$<2 \min$ & $13(16,9 \%)$ & $17(11 \%)$ & \\
\hline$\geq 2 \min$ & $64(83,1 \%)$ & $137(89 \%)$ & $p=0,30$ \\
\hline Saída de Ar Bilateral & $64(83,1 \%)$ & $154(100 \%)$ & $\begin{array}{l}\mathrm{p}<0,001 \\
\text { (Fisher) }\end{array}$ \\
\hline
\end{tabular}

Teste Fisher $\mathrm{p} \leq 0,05$ (significantes)

$<$ menor, $\geq$ maior ou igual, min- minutos

Rev CEFAC, São Paulo, v.9, n.1, 47-54, jan-mar, 2007 
Tabela 3 - Distribuição da amostra segundo o estado nutricional

\begin{tabular}{|c|c|c|c|}
\hline ESTADO NUTRICIONAL & $\begin{array}{c}\text { RESPIRADORES } \\
\text { ORAIS }\end{array}$ & $\begin{array}{c}\text { RESPIRADORES } \\
\text { NASAIS }\end{array}$ & ESTATÍSTICA \\
\hline \multicolumn{4}{|l|}{ IMC } \\
\hline Baixo peso & $3(3,9 \%)$ & $15(9,7 \%)$ & \\
\hline Eutrófico & $54(70,1 \%)$ & $107(69,5 \%)$ & $p=0,24$ \\
\hline Sobrepeso & $20(26 \%)$ & $32(20,8 \%)$ & \\
\hline \multicolumn{4}{|l|}{ ESTATURA/IDADE } \\
\hline$<-2$ escore $z$ & $2(2,6 \%)$ & $1(0,6 \%)$ & \\
\hline$-2--1$ escore $z$ & $3(3,9 \%)$ & $16(10,4 \%)$ & \\
\hline$>-1$ escore $z$ & $72(93,5 \%)$ & $137(89 \%)$ & $p=0,12$ \\
\hline
\end{tabular}

\section{DISCUSSÃO}

O estudo detectou que os respiradores orais apresentavam menor freqüência de saída de ar bilateral à respiração e vedamento labial à mastigação, maior ocorrência de ruído e escape de comida à mastigação, com tendência a redução do paladar e mastigação lenta, em relação aos respiradores nasais. A possibilidade de que crianças com respiração oral podem desenvolver alterações no estado nutricional não foi confirmada no presente estudo, observando-se predominância de indivíduos eutróficos e ausência de diferença significante nas freqüências de crianças com peso baixo e sobrepeso entre os dois grupos.

Um estudo propõe que a obstrução nasal leva à redução do olfato, diminuindo, por conseqüência, o apetite. Olfato e paladar estão intimamente relacionados; o mecanismo do olfato excita os receptores do paladar, influenciando-o, o que pode explicar a ocorrência de desvios nutricionais nos respiradores orais ${ }^{10}$. No presente estudo, não foi observada diferença estatística quanto ao paladar e ao olfato entre os dois grupos. No entanto, é relatada a relação entre esses dois aspectos nos indivíduos com respiração oral, em virtude da não utilização adequada das vias aéreas superiores ${ }^{11}$, sendo comum a queixa de hipogeusia ${ }^{10}$.

A avaliação miofuncional orofacial é importante nos estudos que investigam os aspectos nutricionais, especialmente nas crianças. A observação direta das funções estomatognáticas, como a mastigação, pode revelar dificuldades na alimentação que repercutem diretamente sobre o estado nutricional. Ao abrir a boca para respirar, há adaptações e desequilíbrio das estruturas e funções orofaciais que comprometem a mastigação e a deglutição, e, conseqüentemente, gera dificuldades na alimentação. É importante ressaltar que as alterações das funções estomatognáticas podem iniciar desde a época da transição do aleitamento natural para $\mathrm{o}$ artificial. A maioria das crianças do presente estudo, independentemente do grupo, foi amamentada por um período inferior a quatro meses. O desmame precoce pode levar à ruptura do desenvolvimento motor-oral adequado, provocando alterações na postura e força dos órgãos fonoarticulatórios e prejudicando as funções de mastigação, deglutição, respiração e articulação dos sons da fala. A falta da sucção fisiológica ao peito pode interferir no desenvolvimento motor-oral, possibilitando a instalação de má oclusão, alteração motora-oral e da respiração oral ${ }^{12}$. Nos respiradores orais, a má oclusão (casos de mordida aberta anterior ou cruzada, ausência de elementos dentários anteriores) também é responsável pelo funcionamento inadequado das funções orofaciais e pode causar interferências na mastigação, levando ao predomínio unilateral e modificando o tempo para mastigar ${ }^{13}$.

Cerca de metade dos informantes das crianças com respiração oral relatou o uso de algum líquido durante as refeições e demora no ato de mastigar, confirmando que essas crianças não conseguem comer de boca fechada, não mastigam o suficiente $e$ deglutem ansiosamente 0 alimento quase inteiro ${ }^{3}$. 
Para facilitar a deglutição do alimento, o indivíduo passa a ingerir grande quantidade de líquido à refeição ${ }^{14}$. Observa-se, ainda, que por não conseguir manter a boca fechada, a criança com respiração oral pode associar o ato de alimentar-se à sufocação, podendo ocorrer diminuição da quantidade de alimento ingerido, tornando-a mais emagrecida ou, por outro lado, a ingestão de alimentos pastosos, de deglutição mais fácil e, em geral, de alto teor calórico, predispõe ao excesso de peso. Assim, modificações compensatórias na mastigação e na respiração podem causar alterações nutricionais no respirador oral ${ }^{3}$.

Os resultados da presente pesquisa mostraram que as crianças do grupo de respiradores orais apresentaram maior ocorrência de mastigação ruidosa quando comparadas com as crianças respiradoras nasais. Isso pode acontecer porque a cavidade oral é uma via na qual ocorrem as funções de mastigar e respirar simultaneamente. Dessa forma, para poder deglutir, a criança pára a mastigação, faz o estalo na boca enquanto o ar entra e, só então, engole o alimento ${ }^{15}$.

Outro aspecto que merece destaque nos resultados obtidos é a identificação de escape de comida e menor possibilidade de vedamento labial, com diferença estatística entre os dois grupos. Essas características presentes no grupo de respiradores orais podem estar associadas ao funcionamento inadequado do sistema estomatognático, em especial à hipofunção da musculatura orofacial - o funcionamento inadequado dos lábios e das bochechas pode alterar a mastigação, tornando-a ineficiente.

Observa-se que não houve diferença significante nos padrões de lado mastigatório entre os grupos, porém, a mastigação predominantemente bilateral alternada ocorreu em apenas pouco mais da metade das crianças respiradoras orais. Esse padrão mastigatório depende da adequação do crescimento e do desenvolvimento craniofacial, do equilíbrio oclusal e da maturação neuromuscular. O respirador oral geralmente não apresenta tal equilíbrio morfofuncional, o que pode justificar as alterações encontradas na mastigação $^{16}$.

Não foram encontrados estudos que demonstrem relação direta entre respiração oral e alterações de peso e estatura nas crianças, porém, um estudo observou que a doença alérgica isolada não foi a causa da baixa estatura, mas o nível socioeconômico desfavorável potencializou o retardo do crescimento ${ }^{17}$. Paralelamente, pesquisadores detectaram freqüência maior de baixa estatura nos pacientes com rinite alérgica, concluindo que a doença por si só promove déficit de crescimento ${ }^{18}$. Foi observado em uma pesquisa que algumas crianças alérgicas apresentam parada do crescimento que se manifesta inicialmente por perda de peso, e que, com a persistência dos sintomas podem ter comprometimento de sua esta- tura e maturidade óssea ${ }^{19}$. Outros autores demonstraram que o tratamento da obstrução nasal por tonsilectomia e adenoidectomia nas crianças respiradoras orais pode melhorar o desenvolvimento pôndero-estatural ${ }^{20,21}$.

As avaliações da aeração nasal e da função mastigatória são realizadas na prática fonoaudiológica, porém a dificuldade na padronização de protocolos pode interferir na obtenção dos resultados. A avaliação da função nasal pelo método do espelho, além da subjetividade, mede o ar expirado, porém os pacientes com obstrução de vias aéreas superiores têm dificuldade principalmente na inspiração ${ }^{21}$. A escolha do pão, observação direta e cronometragem para avaliação da mastigação, embora baseados em protocolos existentes na literatura ${ }^{6,7,16}$, também apresentam subjetividade. Deve-se salientar que o fato de todas as crianças terem sido avaliadas em um único encontro e em momento não habitual de alimentação pode modificar o padrão da função mastigatória. Métodos que padronizem a avaliação da mastigação devem ser propostos e validados na busca de resultados mais objetivos.

As crianças respiradoras orais apresentam vários fatores que as tornam mais predispostas às alterações nutricionais do que aquelas respiradoras nasais, conforme abordado anteriormente, porém, isso não foi detectado neste estudo. As crianças estudadas estavam em acompanhamento médico e o possível controle da rinite pode ter reduzido o impacto da respiração oral sobre o estado nutricional. Ressalta-se que, além da ingestão alimentar, o gasto energético com atividades físicas também influencia o estado nutricional e ambos estão na dependência de fatores socioeconômicos e culturais. Pode-se especular que os respiradores orais teriam maior dificuldade durante a realização de exercícios físicos, impossibilitando maior gasto da energia consumida. Portanto, a avaliação de fatores como atividade física e ingestão alimentar podem auxiliar a esclarecer as repercussões nutricionais decorrentes da respiração oral e devem ser considerados em estudos posteriores.

Estudos longitudinais, com o objetivo de investigar maiores números de indivíduos e analisar o estado nutricional de crianças respiradoras orais, em diferentes faixas etárias, bem como a comparação de diversas etiologias da respiração oral, poderão oferecer maior sustentação teórica a este tema.

Mesmo havendo indícios que crianças que respiram pela boca podem apresentar alteração no estado nutricional, estes dados não foram totalmente elucidados na presente pesquisa. Porém foi possível lançar o olhar para as repercussões que não estão apenas direcionadas à boca do indivíduo impossibilitado de respirar pelo nariz.

Este trabalho aponta para a necessidade de aprofundar ainda mais os estudos sobre repercus- 
sões mais globais que acometem os respiradores orais e mostra que no trabalho como clínicos e pesquisadores é imprescindível a busca da melhoria da qualidade de vida dessas crianças.

\section{CONCLUSÃO}

Com base no presente estudo comparativo entre o grupo de crianças respiradoras nasais e respiradoras orais, foi possível verificar que houve diminuição da ingestão de carboidratos e aumento da ingestão de lipídeos em crianças respiradoras orais com baixo peso e eutróficas quando comparadas às crianças respiradoras nasais com o mesmo estado nutricional; dados estes que alertam para as possíveis repercussões da respiração oral no estado nutricional.

As crianças respiradoras orais apresentaram maiores alterações na mastigação quando comparadas às crianças respiradoras nasais, o que não aconteceu para o olfato e paladar. A avaliação da aeração nasal apresentou saída bilateral prevalente nos dois grupos avaliados, embora houvesse uma maior ocorrência de sintomas de ronco e escape de saliva durante o sono, no grupo de crianças respiradoras orais.

\section{ABSTRACT}

Purpose: identify nutritional state, masticatory function, respiratory mode, sense of smell and sense of taste among mouth breathing children. Methods: nasal aeration, mastication, nutritional state of 77 mouth breathing children and 154 nasal breathers children between six and ten years were evaluated. Results: mouth breathers exhibited alterations in the masticatory pattern when compared to the nasalbreathers, but did not have statistical significance in nutritional state. Conclusion: the oral-breathing children had more alteration in sleep, reduction in the ingestion of carbohydrate and increase in ingestion of lipids, however, no consistent data were found pointing out the association between mouth breathing and nutritional condition.

KEYWORDS: Mouth Breathing; Nutritional Status; Speech, Language and Hearing Sciences

\section{REFERÊNCIAS}

1.Weckx LLM, Weckx LY. Respirador bucal: causas e conseqüências. Rev Bras Med. 1995; 52:863-74. 2.Montonaga SM, Berti LC, Anselmo-Lima WT. Respiração bucal: causas e alterações no sistema estomatognático. Rev Bras Otorrinolaringol. 2000; 66(4):373-9.

3.Carvalho GD. Alterações alimentares e do apetite. In: Carvalho GD. S.O.S. respirador bucal. São Paulo: Lovise; 2003. p. 137-44.

4.Subtelny JD. Effect of diseases of tonsils and adenoids on dentofacial morphology. Ann Otol Rhinol Laryngol. 1975; 84(2):50-4.

5.Di Francesco RC, Passeroti G, Paulucc B, Miniti A. Respiração oral na criança: repercussões diferentes de acordo com o diagnóstico. Rev Bras Otorrinolaringol. 2004; 7(5):665-70.

6. Marchesan IQ. Avaliação e terapia dos problemas da respiração. In: Marchesan IQ. Fundamentos em fonoaudiologia: aspectos clínicos da motricidade oral. Rio de Janeiro: Guanabara Koogan; 1998. p. 23-36. 7.Junqueira P. Avaliação miofuncional. In: Marchesan IQ. Fundamentos em fonoaudiologia: aspectos clínicos da motricidade oral. Rio de Janeiro: Guanabara Koogan; 1998. p. 12-21.

8.Marchesan IQ. Protocolo de avaliação miofuncional orofacial. In: Krakauer HL, Di Francesco RC, Marchesan IQ, organizadores. Respiração oral. Coleção CEFAC. São José dos Campos: Pulso; 2003. p. 55-79.

9. Motta MEFA, Silva GAP. Desnutrição e obesidade em crianças: delineamento do perfil de uma comunidade de baixa renda. J Pediatr. 2001; 77(4):288-93. 10. Klein E. Obstrução nasal: um obstáculo à vida. Rev Bras Otorrinolaringol. 1987; 53(4):106-10.

11. Ferraz MC. Manual prático de deglutição atípica e problemas correlatos: terapia miofuncional nos tratamentos orofaciais. Rio de Janeiro: Revinter; 1996. 12. Neiva FCR, Cattoni DM, Ramos JL, Issler H. Desmame precoce: implicações para o desenvolvimento motor-oral. J Pediatr. 2003; 79(1):7-12.

13. Freitas V, Matsumoto MAN. Incidência de más oclusões dentárias em pacientes respiradores bucais. In: Marchesan IQ, Zorzi JL. Tópicos em fonoaudiologia. São Paulo: Revinter; 2003. p. 279-87.

14. Farronato GP, Gianni E. Función respiratoria y su repercusión estomatognática y orgánica. Ortodoncia. 1997;61(121):61-76.

15. Tomé MC, Marchiori SC, Pimentel R. Mastigação: implicações na dieta do respirador bucal. J Bras Fonoaudiol. 2000; 1(3):60-5. 
16. Bianchini EMG. Mastigação e ATM: avaliação e terapia. In: Marchesan IQ. Fundamentos em fonoaudiologia: aspectos clínicos da motricidade oral. Rio de Janeiro: Guanabara Koogan; 1998. p. 37-49. 17. Solé DN, Scalabrin DMF, Sano FM, Marcia C, Naspitz CK, Castro-Spinola AM, Denardin O. Doença alérgica e sua repercussão sobre o crescimento. J Pediatr. 1991; 67(3):92-100.

18. Sant'anna CA, Solé DN, Charles K. Alergia respiratória e baixa estatura em crianças: estudo da taxa de crescimento. Rev Bras Alerg Imunopatol. 1996;
19(1):6-13.

19. Cohen MB, Abram LE. Growth patterns of allergic children. J Allergy. 1948; 19:165-7.

20. Soultan Z, Wadowski S, Rao M, Kravath RE. Effect of treating obstructive sleep apnea by tonsillectomy and/or adenoidectomy on obesity in children. Arch Pediatr Adolesc Med. 1999; 153(1):33-7.

21.Di Francesco RC. Avaliação otorrinolaringológica da respiração oral. In: Krakauer LH, Di Francesco RC, Marchesan IQ. Respiração oral: abordagem interdisciplinar. São José dos Campos: Pulso; 2003. p. 43-5.

RECEBIDO EM: 18/12/06

ACEITO EM: 21/02/07

Endereço Para Correspondência:

Avenida Norte, $\mathrm{n}^{\circ} 8042$

Recife - PE

CEP: 52091-000

Tel: (81) 21018335

E-mail:danyfono@fir.br

Rev CEFAC, São Paulo, v.9, n.1, 47-54, jan-mar, 2007 